POSTER SESSION 


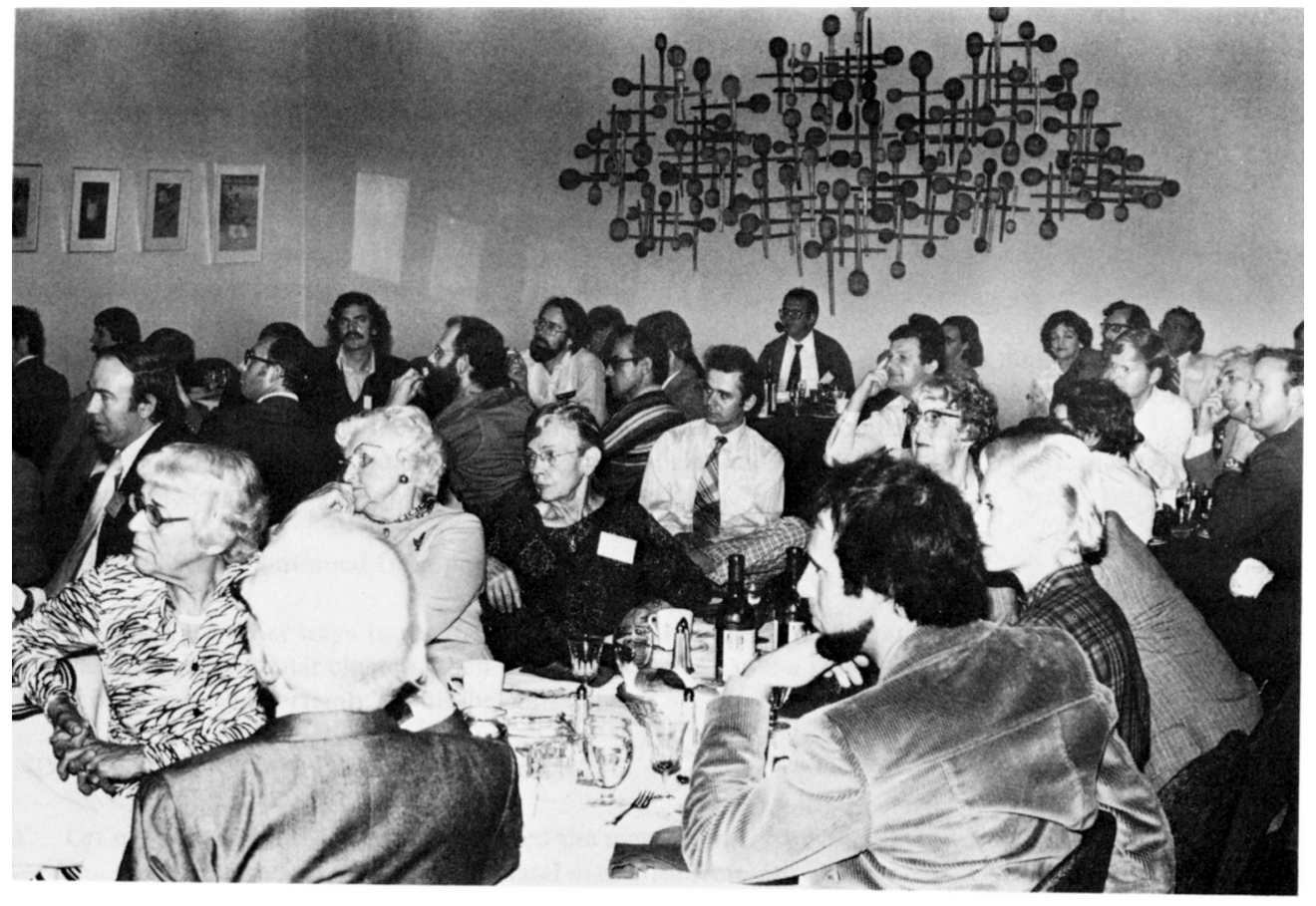

The Banquet on November 4, 1977. 


\title{
A REVISED CLASSIFICATION SYSTEM IN THE RED FOR S STARS
}

\author{
Thomas B. Ake \\ Warner and Swasey Observatory, \\ Case Western Reserve University
}

\section{ABSTRACT}

A classification scheme in the red spectral region for the $\mathrm{S}$ stars is described. For those stars exhibiting both $\mathrm{ZrO}$ and TiO bands, a modified version of the Keenan (1954) system is used for the assignment of temperature subtypes. For the pure S stars, a new system is introduced utilizing $\mathrm{ZrO}$ band strengths and the sodium D lines. Comparisons between the revised types and photometric colors have been made. In addition a new abundance index is proposed based on the relative strength of YO compared to ZrO and TiO. The revised system provides a connecting link between the $M$ and $C$ systems so that all red giants may be classified consistently from the strengths of $\mathrm{TiO}, \mathrm{ZrO}$ and $\mathrm{Na} \mathrm{D}$.

The classification of the $S$ stars is greatly complicated by the variety of S-type hybrids that have been found to exist. Although the $\mathrm{S}$ stars are defined by the presence at low dispersion of $\mathrm{ZrO}$ bands, some members of the class are similar to the $M$ giants by exhibiting strong TiO, while others are weak-banded and display atomic-line spectra resembling carbon stars. Presumably, as a class the $S$ stars are chemically intermediate between the $M$ giants and the carbon stars, and thus, are difficult to classify because their molecular bands are severely affected by heavy-element and $\mathrm{C} / \mathrm{O}$ abundance anomalies. The classification system devised by Keenan (1954) handles this problem by assigning temperature classes according to a weighted sum of $\mathrm{ZrO}$ and $\mathrm{TiO}$ band strengths such that the stronger bands are weighted more heavily. He also calculates an abundance parameter based on the $\mathrm{ZrO} / \mathrm{TiO}$ ratio, although Keenan and McNeil (1976) have eliminated this index in the S notation and have replaced it by 1 isting the TiO and $\mathrm{ZrO}$ strengths individually 
with the temperature class assignment. The Keenan system, however, is inappropriate for the pure $\mathrm{S}$ and SC stars because TiO is absent and there is 1ittle similarity to the M giants. Because the $\mathrm{C}$ system for the carbon stars relies upon atomic-line criteria for the temperature types, it seems reasonable to assume that the same criteria should be available for the pure S and SC types. At low dispersion in the red, the $\mathrm{Na} \mathrm{D}$ lines are quite useful, and a revised S-type system is introduced to improve the spectral types of the stars with Keenan abundance classes 8 and 9.

Image-tube spectrograms taken with the Warner and Swasey 91-cm reflector have been obtained for most of Keenan's original S stars and for some additional objects found in Stephenson's (1976) S star catalogue. The region 5450-7000 $\AA$ is observed at an inverse dispersion of $105 \AA / \mathrm{mm}$ with an estimated resolution of $5 \AA$. For the pure $S$ stars, the line spectra are visible but indistinct on these plates, and only the D lines were found to be use$\mathrm{ful}$ as an atomic-line classification criterion. Absolute band strengths of TiO and ZrO were visually assigned on the Keenan systems, and $\mathrm{Na} \mathrm{D}$ intensities were measured on a new system. The Dline system was later compared with that of the $C$ stars so that the SC stars could be translated to comparable $C$ types. It was found that an $S$ star with $D=1$ corresponds to type $C 5$.

For the stars with strong TiO, Keenan's system is retained except for a minor change in the zero-point of the scale. Whereas the Keenan TiO strengths are the equivalent $M$ type +1 , the TiO intensity here is taken to be just the $M$ type in order to accommodate the higher dissociation energy of ZrO compared to TiO. When proceeding from the $\mathrm{K}$ giants to the $\mathrm{S}$ stars, ZrO should appear before TiO, and adding 1 to the $M$ types would exaggerate the coolness of the stars. For the pure S stars, TiO is replaced by $\mathrm{Na} \mathrm{D}$ in the computation. Since $C$ types can be assigned by the $D$ lines alone (Yamashita 1972), this substitution carries the S system to the $\mathrm{C}$ system for the weak-banded pure S and SC stars, where the $\mathrm{D}-1$ ine intensity is greater than the $\mathrm{ZrO}$ strength.

The classification then is

$\mathrm{S}$-type $=$ the stronger of $\mathrm{ZrO}$ and $\mathrm{TiO}+1 / 2$ the weaker of these

or

$\mathrm{S}$-type $=$ the stronger of $\mathrm{ZrO}$ and $\mathrm{Na} \mathrm{D}+1 / 2$ the weaker of these.

For most stars there is little ambiguity about which criteria to use for the classification since the D lines are masked by TiO $5849 \AA$ when Til is strong. The warmer members of the class, however, may display $\mathrm{TiO}$ and $\mathrm{Na} \mathrm{D}$ at the same time so that the spectral type is assigned at the discretion of the observer. Two cool 
stars, AA Cyg and BI And, are unusual in that both TiO and $\mathrm{Na} D$ are quite strong, but $\mathrm{TiO} \sim \mathrm{Na} \mathrm{D}$ so that the spectral types are stable.

Although different criteria are used for the Ti0-strong and pure $S$ types, comparisons of the new types with Piccirillo's (private communication) eight-color photometry of non-Mira S stars indicates that the types are assigned on a common temperature scale. The major improvement of the revised system is that the pure $S$ stars are brought in line with the other S types. The most discrepant star is SU Mon, whose super-saturated D lines are comparable to those of the D-line carbon stars such as WZ Cas. Although some $S$ stars display $D$ lines approaching the intensity found in SU Mon, unlike this star their entire spectra indicate extremely low temperatures. For the long-period variables, the types appear to be well-behaved with phase.

Furthermore observations of the red Yo bands were found to be inadequately explained by temperature differences between the various $S$ types. The peculiar behavior of $Y O$ in $M$ giants and $S$ stars is well-known (e.g. Keenan 1966). These bands begin to appear in some $M$ giants and are quite strong even at classification dispersion for the $S$ stars. Their appearance in the $M$ giants has been attributed to both incipient s-processing of $Y$ as well as increasing $\mathrm{C} / 0$ from the $\mathrm{M}$ giant to the $\mathrm{S}$ stage. In $\mathrm{S}$ stars of the same temperature class, YO appears weakly when TiO $\gg \mathrm{ZrO}$ or $\mathrm{ZrO}$ $\gg \mathrm{TiO}$, and is strongest when $\mathrm{ZrO} \sim \mathrm{TiO}$. For the $\mathrm{SC}$ stars, $\mathrm{ZrO}$ is present with strength less than 0.5 , and $Y O$ and TiO are absent. It appears that the $\mathrm{S}$ stars can be divided into six abundance classes based on the comparison of $\mathrm{YO}$ to TiO and $\mathrm{ZrO}$ ( $\mathrm{Table} \mathrm{I}$ ). The YO strengths are multiplied by 2 since they are not as strong as TiO and ZrO on these plates.

This pattern of behavior is predicted by increasing $\mathrm{C} / \mathrm{O}$ from the TiO-strong stage to the pure S and SC types (e.g. Scalo and Ross 1976). Because the dissociation potential of YO lies between

\section{TABLE I}

DEFINITION OF THE ABUNDANCE INDEX

\begin{tabular}{|c|l|}
\hline Index & Spectral Characteristics \\
\hline 1 & TiO $>\mathrm{ZrO}$ and YO \\
2 & $\mathrm{TiO} \geq \mathrm{ZrO} \geq 2 \times \mathrm{YO}$ \\
3 & $2 \times \mathrm{YO} \geq \mathrm{ZrO} \geq \mathrm{TiO}$ \\
4 & $\mathrm{ZrO} \geq 2 \mathrm{x} \mathrm{YO}>\mathrm{TiO}$ \\
5 & $\mathrm{ZrO} \geq 2 \times \mathrm{YO} ; \mathrm{TiO}=0$ \\
6 & ZrO weak; YO, TiO $=0$ \\
\hline
\end{tabular}


that of $\mathrm{TiO}$ and $\mathrm{ZrO}$, and $\mathrm{YO}$ is depleted by dioxide formation unlike $\mathrm{ZrO}$, YO should have its highest concentration when the free-oxygen supply is small enough to reduce the $\mathrm{TiO}$ and $\mathrm{YO}_{2}$ concentrations, but not so small that the $\mathrm{ZrO}$ content is reduced too. Although the behavior of $\mathrm{YO}$ is more complicated than this simple picture (Piccirillo, private communication), it is suggested that these crude divisions can be used as an abundance index. This parameter appears to be related to the $\mathrm{C} / 0$ ratio, but because $\mathrm{s}$-process enhancements influence the appearance of $\mathrm{ZrO}$ and $\mathrm{YO}$, it should not be labelled as a $\mathrm{C} / 0$ index. In particular, stars in abundance classes 2 and 3, where all molecular bands are strong, may have increased heavy-element abundances without $\mathrm{C} / 0$ increases. It is a well-known fact that both $s$-process enhancements and $\mathrm{c} / 0$ variations must be invoked simultaneously to explain both the molecular-band and atomic-line spectra of the S stars.

\section{ACKNOWLEDGMENTS}

The writer is pleased to acknowledge suggestions by John Piccirillo, William P. Bidelman and Philip C. Keenan, and is especially indebted to John Piccirillo for the use of his photometric observations prior to publication.

\section{REFERENCES}

Keenan, P.C. (1954). Astrophys. J., 120, 484. - (1966). in IAU Symposium No. 24, Spectral Classification and Multicolor Photometry, K. Lodén, L. O. Lodén, and U. Sinnerstad, eds., Academic Press, New York, p. 26. , and McNeil, R. C. (1976). An Atlas of Spectra of the Cooler Stars, The Ohio State University Press, Columbus.

Scalo, J. M., and Ross, J. E. (1976). Astron. Astrophys., 48, 219. Stephenson, C. B. (1976). Publ. Warner and Swasey Observatory, Vo1. 1, No. 4 .

Yamashita, Y. (1972). Ann. Tokyo Astron. Obs., 2nd Series, XIII, No. 3. 